Original Research Article

\title{
A comparative study of efficacy and tolerability of doxofylline over other methylxanthenes in mild to moderate persistent bronchial asthma patients in a tertiary care hospital
}

\author{
Satyendra K. Alladi*, Imran Khan
}

Department of Pharmacology, Kakatiya Medical College, Warangal, Telangana, India

Received: 30 June 2018 Accepted: 27 July 2018

\section{*Correspondence to:} Dr. Satyendra K. Alladi, Email: askvijpaniram99@ gmail.com

Copyright: (C) the author(s), publisher and licensee Medip Academy. This is an openaccess article distributed under the terms of the Creative Commons Attribution NonCommercial License, which permits unrestricted noncommercial use, distribution, and reproduction in any medium, provided the original work is properly cited.

\begin{abstract}
Background: Studies have shown that doxofylline is endowed with a remarkable bronchodilator activity with less extra respiratory effects than other methylxanthines. To compare the efficacy and tolerability of doxofylline over other methylxanthines in mild to moderate persistent bronchial Asthma patients. Methods: It is a comparative study conducted in patients of mild to moderate persistent bronchial Asthma in the outpatient department of regional chest hospital. The subjects were randomly assigned into 2 groups, one group treated with oral doxofylline and the other with theophylline oral or Deriphylline. Efficacy was measured objectively using spirometric parameters like FEV1 (Forced Expiratory Volume at the end of 1 second), FVC (Forced Vital Capacity), FEV1/FVC and PEFR (Peak Expiratory flow Rate), Subjectively by the Asthma control test questionnaire, subjective rating of Asthma control and the need for use of rescue medications like $\beta 2$ agonists in the previous four weeks. Tolerability were done at base line and at the end of study.

Results: Doxofylline compared to other methylxanthines groups was statistically better with respect to subjective parameters like Asthma control tests questionnaire, subjective rating of Asthma control. Doxofylline had equal efficacy with spirometric parameters compared to other methylxanthines. Doxofylline was significantly better tolerated with adverse drug reactions noted in $10 \%$ compared to $28 \%$ in other methylxanthins group.

Conclusions: Doxofylline with better tolerability profile and equivalent efficacy seemed to be a good alternative to other methylxanthines in the treatment of bronchial Asthma.
\end{abstract}

Keywords: Bronchial asthma, Doxofylline, Efficacy, Methylxanthines, Tolerability

\section{INTRODUCTION}

Bronchial asthma is a chronic inflammatory disorder characterized by airway hyper responsiveness that leads to recurrent episodes of wheezing, breathlessness, chest tightness, and coughing particularly at the night or early morning. Often associated with variable, widespread obstruction that is reversible either spontaneously or with treatment. $^{1,2}$
Asthma is prevalent worldwide affecting more than 300 million. ${ }^{3}$ Global prevalence ranges from 1 to $18 \% .^{1}$ India has approximately 15-20 million asthmatics.

Asthma management is to achieve and maintain clinical control. Effective control of acute attacks; and in the long term, prevention of further episodes and airway remodelling at an early stage. Medications to treat asthma classified as controllers and relievers. Controllers, taken daily for clinical control of asthma by its anti inflammatory 
effects. They include inhaled and systemic corticosteroids, theophylline. Cromones and anti-Ig E. Relievers, medications used as needed basis, to reverse bronchoconstriction and relieve symptoms. They include rapid acting inhaled $\beta_{2}$ agonists, inhaled anticholinergics, short acting theophylline, and short acting oral $\beta_{2}$ agonists. ${ }^{1,2}$

Methylxanthines constitute non-selective phosphodiesterase inhibitors. Characterized by mild bronchodilator, immunomodulator, anti-inflammatory, bronchoprotective, mucoregulatory, inflammatory cell stabilizing and steroid sparing properties. ${ }^{3,4}$

Theophylline, a methylxanthine with narrow therapeutic index, often results in wide range of adverse effect of cardiac, gastro - intestinal and central nervous systems accounting for the poor compliance and high dropout rates. Doxofylline [7-(1,3-dioxolane-2-methyl1) theophylline] has equal or superior bronchodilator activity than theophylline and better safety profile due to reduced affinity for adenosine $A_{1}$ and $A_{2}$ receptors. It ameliorates spirometric parameters, reduces attack rates and the need for $\beta 2$ agonists. $^{4}$

Pharmacotherapy forms a major role in both treatment and control of asthma. Hence, there is constant search for new drugs with better efficacy and reduced side effect profile. Cochrane's group concludes xanthines as first choice therapy to prevent and relieve symptoms in asthma and to reduce the requirements of rescue medication in mild to moderate asthma. ${ }^{4}$ Present study is aimed to compare the efficacy and tolerability of doxofylline over other methylxanthines in mild to moderate persistent bronchial asthma patients.

\section{METHODS}

It was a single center, prospective comparative study, period of study was from January 2015 to September 2015.

\section{Study population}

Patients with mild to moderate persistent bronchial asthma attending outpatient department of chest hospital, study was started after the protocol was approved by the Institutional Ethics Committee. All the patients diagnosed with mild to moderate persistent Bronchial Asthma were screened and selected based on following criteria.

\section{Inclusion criteria}

- $\quad$ Patients giving informed consent

- Patients aged between 18-65 years of either sex.

- Patients clinically diagnosed with mild to moderate persistent bronchial asthma.

- $\quad$ Patients with FEV1 (Forced expiratory volume in the first second of expiration)

- Value of $50 \%$ or more of predicted.
- Improvement in FEV1 greater than $12 \%$ after bronchodilator inhalation.

- Patients on inhaled corticosteroids.

\section{Exclusion criteria}

Major respiratory illness other than asthma like Chronic obstructive pulmonary disease or other relevant lung disease causing alternative impairment in lung function.

- $\quad$ Patients with co-morbid conditions - Ischemic heart disease, congestive cardiac failure, renal and hepatic dysfunction, neurological, other endocrinal and hematological abnormalities.

- $\quad$ Patients unwilling to comply with the requirement of the protocol.

- $\quad$ Smokers, pregnant, and lactating women.

- History of known allergy/intolerance/hyper sensitivity to study drugs.

- $\quad$ Patients on regular treatment with drugs that interact with methylxanthines.

- Patients on systemic corticosteroids.

- Patients with Class III obesity according to Indian guidelines for obesity.

The study subjects giving informed consent, fulfilling the inclusion/exclusion criteria were assigned into 2 groups of 30 patients each.

- Group 1: Patients treated with doxofylline 400mg once a day.

- $\quad$ Group 2: Patients treated with other methylxanthines like Theophylline 200mg SR once a day Deriphylline 150mg SR two times a day.

Assessment was done by measuring the efficacy parameters like pulmonary Function Test (Spirometry) FVC, FEV1, FEV1/FVC and PEFR.

The asthma control questionnaire: five items-shortness of breath, patient rating of control, use of rescue medication, work/school limitations related to asthma and nocturnal asthma symptoms, each of the five items assessed on a 5point scale and the response is summed to give scores ranging from 5 (poor control) to 25 (complete control).

Tolerability was assessed by:

- Adverse drug reactions reported voluntarily by the patients, observed or enquired were recorded.

A baseline measurement of spirometry parameters was done, and both the groups were followed up at 4, 8, and 12 weeks for the outcome measurement.

\section{Statistical analysis}

The data obtained was analyzed using the SPSS software version 22. Descriptive statistics including the mean and 
standard deviation were calculated for quantitative variables. Quantitative variables were compared with Chisquare test, Fisher exact test, Student's t-test. Charts were generated using MS-EXCEL and MS -WORD.

\section{RESULTS}

Totally 60 patients suffering from mild to moderate persistent bronchial asthma patients were evaluated and analyzed for epidemiological profile, disease spectrum, efficacy and tolerability parameter.

\section{- DOXOFYLLINE $\square$ OTHERMETHYLXANTHINES}

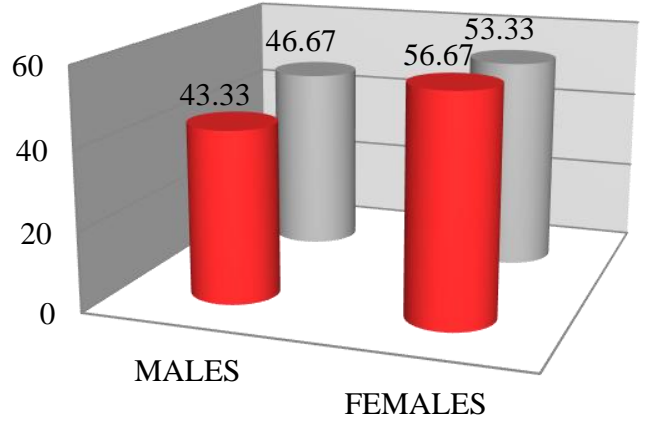

Figure 1: Gender wise distribution of patients studied.

In the present study, majority of the patients were females $56.6 \%$ and $53.3 \%$ compared to males $43.33 \%$ and $46.67 \%$ in doxofylline and other methylxanthines group respectively.

\section{- DOXOFYLLINE —OTHER METHYLXANTHINES}

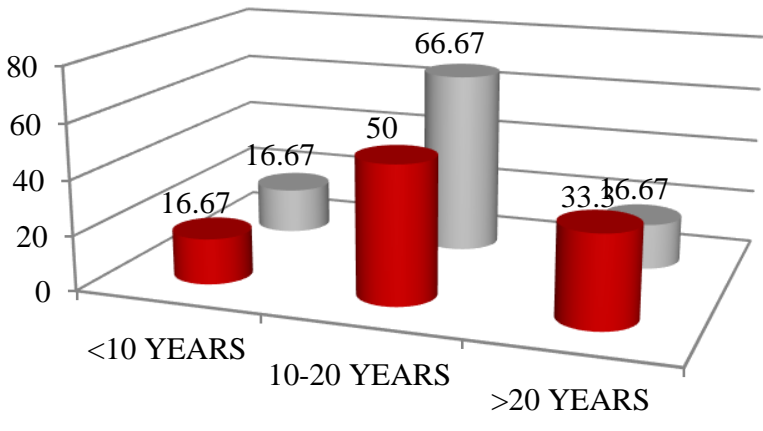

Figure 2: Duration of illness (years) in the two groups.

Mean duration of illness was 17.5 and 15.17 years in doxofylline and the other methylxanthines group respectively.

The asthma control test questionnaire revealed statistically significant improvement in both the groups compared to the baseline from $2^{\text {nd }}$ visit onwards. There was significant difference in the doxofylline group compared to other methylxanthines group throughout the study.

Table 1: Comparison of efficacy parameters in two groups of patients studied (Asthma control test questionnaire score).

\begin{tabular}{|c|c|c|c|}
\hline \multicolumn{4}{|c|}{ Asthma control test questionnaire score } \\
\hline & Doxofylline & Othermethylxanthines & P value \\
\hline Baseline & $16.82 \pm 1.44$ & $15.65 \pm 0.58$ & $<0.001 * *$ \\
\hline $1^{\text {st }}$ Visit & $16.9 \pm 1.25$ & $16.44 \pm 0.68$ & $0.047 *$ \\
\hline $2^{\text {nd }}$ Visit & $17.64 \pm 1.22$ & $16.89 \pm 0.68$ & $0.001 * *$ \\
\hline $3^{\text {rd }}$ Visit & $18.6 \pm 1.07$ & $18.00 \pm 0.80$ & $0.005 * *$ \\
\hline \multicolumn{4}{|c|}{ Significance from base line } \\
\hline $1^{\text {st }}$ Visit & $\Delta=0.08 ; \mathrm{P}=0.351$ & $\Delta=0.78 ; \mathrm{P}=0.001 * *$ & - \\
\hline $2^{\text {nd }}$ Visit & $\Delta=0.82 ; \mathrm{P}=0.001 * *$ & $\Delta=1.23 ; \mathrm{P}<0.001 * *$ & - \\
\hline $3^{\text {rd }}$ Visit & $\Delta=1.72 ; \mathrm{P}=0.001 * *$ & $\Delta=2.34 ; \mathrm{P}<0.001 * *$ & - \\
\hline
\end{tabular}

Table 2: Comparison of subjective rating of asthma control.

\begin{tabular}{|c|c|c|c|}
\hline \multicolumn{4}{|c|}{ Subjective rating of asthma control } \\
\hline Efficacy parameters & Doxofylline & Other methylxanthines & P value \\
\hline Baseline & $58.22 \pm 3.11$ & $56.97 \pm 5.00$ & 0.155 \\
\hline $1^{\text {st }}$ Visit & $61.72 \pm 2.51$ & $63.02 \pm 2.73$ & $0.022 *$ \\
\hline $2^{\text {nd }}$ Visit & $65.64 \pm 1.66$ & $66.44 \pm 3.05$ & 0.116 \\
\hline $3^{\text {rd }}$ Visit & $73.26 \pm 2.35$ & $72.23 \pm 2.51$ & $0.053 *$ \\
\hline \multicolumn{4}{|c|}{ Significance from base line } \\
\hline $1^{\text {st }}$ Visit & $\Delta_{=} 3.50 ; \mathrm{P}<0.001 * *$ & $\Delta=6.05 ; \mathrm{P}=0.001 * *$ & - \\
\hline $2^{\text {nd }}$ Visit & $\Delta=7.42 ; \mathrm{P}<0.001 * *$ & $\Delta=9.47 ; \mathrm{P}<0.001 * *$ & - \\
\hline $3^{\text {rd }}$ Visit & $\Delta=15.04 ; \mathrm{P}<0.001 * *$ & $\Delta=15.26 ; \mathrm{P}<0.001 * *$ & - \\
\hline
\end{tabular}


Table 3: Comparison of forced vital capacity in the two groups of patients studied.

\begin{tabular}{|llll|}
\hline Forced vital capacity & Doxofylline & Othermethylxanthines & P value \\
\hline Baseline & $2.58 \pm 0.46$ & $2.72 \pm 0.44$ & 0.136 \\
\hline \% Predicted of baseline & $79.85 \pm 3.25$ & $80.34 \pm 1.92$ & 0.378 \\
\hline $1^{\text {st }}$ Visit & $2.64 \pm 0.47$ & $2.78 \pm 0.45$ & 0.132 \\
\hline \% Predicted of $1^{\text {st }}$ Visit & $81.64 \pm 3.13$ & $82.20 \pm 1.93$ & 0.303 \\
\hline $2^{\text {nd }}$ Visit & $7.57 \pm 34.55$ & $2.82 \pm 0.46$ & 0.370 \\
\hline$\%$ Predicted of $2^{\text {nd }}$ Visit & $83.06 \pm 2.99$ & $83.34 \pm 2.02$ & 0.593 \\
\hline $3^{\text {rd }}$ Visit & $2.71 \pm 0.47$ & $2.84 \pm 0.46$ & 0.186 \\
\hline$\%$ Predicted of $3^{\text {rd }}$ Visit & $83.96 \pm 2.96$ & $83.81 \pm 1.88$ & 0.781 \\
\hline Significance from base line & & & - \\
\hline $1^{\text {st }}$ Visit & $\Delta=0.056 ; \mathrm{P}<0.001 * *$ & $\Delta=0.06 ; \mathrm{P}=0.001 * *$ & - \\
\hline $2^{\text {nd }}$ Visit & $\Delta=0.102 ; \mathrm{P}<0.001 * *$ & $\Delta=0.10 ; \mathrm{P}<0.001 * *$ & - \\
\hline $3^{\text {rd }}$ Visit & $\Delta=0.129 ; \mathrm{P}<0.001 * *$ & $\Delta=0.11 ; \mathrm{P}<0.001 * *$ & - \\
\hline
\end{tabular}

Table 4: Comparison of forced expiratory volume at end of 1 second (FEV1) in the two groups of patients studied.

\begin{tabular}{|c|c|c|c|}
\hline & Doxofylline & Othermethylxanthines & P value \\
\hline Baseline & $1.74 \pm 0.32$ & $1.83 \pm 0.29$ & 0.124 \\
\hline$\%$ Predicted of baseline & $64.1 \pm 3.29$ & $64.06 \pm 2.65$ & 0.955 \\
\hline Post salbutamol & $2.17 \pm 0.39$ & $2.34 \pm 0.40$ & $0.040^{*}$ \\
\hline$\%$ Predicted & $80.04 \pm 3.31$ & $81.27 \pm 3.63$ & $0.089+$ \\
\hline $1^{\text {st }}$ Visit & $1.86 \pm 0.34$ & $1.95 \pm 0.32$ & 0.169 \\
\hline$\%$ Predicted of $1^{\text {st }}$ Visit & $68.26 \pm 3.29$ & $68.95 \pm 2.74$ & 0.630 \\
\hline $2^{\text {nd }}$ Visit & $1.97 \pm 0.35$ & $2.06 \pm 0.34$ & 0.192 \\
\hline$\%$ Predicted of $2^{\text {nd }}$ Visit & $72.58 \pm 3.2$ & $71.97 \pm 2.89$ & 0.347 \\
\hline $3^{\text {rd }}$ Visit & $2.1 \pm 0.39$ & $2.19 \pm 0.36$ & 0.205 \\
\hline$\%$ Predicted of $3^{\text {rd }}$ Visit & $76.82 \pm 3.24$ & $76.30 \pm 3.00$ & 0.429 \\
\hline \multicolumn{4}{|l|}{ Significance from base line } \\
\hline $1^{\text {st }}$ Visit & $\Delta=0.43 ; \mathrm{P}<0.001 * *$ & $=0.504 ; \mathrm{P}=0.001 * *$ & - \\
\hline $2^{\text {nd }}$ Visit & $\Delta=0.119 ; \mathrm{P}<0.001 * *$ & $=0.114 ; \mathrm{P}<0.001 * *$ & - \\
\hline $3^{\text {rd }}$ Visit & $\Delta=0.235 ; \mathrm{P}<0.001 * *$ & $\Delta=0.229 ; \mathrm{P}<0.001 * *$ & - \\
\hline
\end{tabular}

The Subjective rating of asthma control revealed statistically significant improvement in both the groups compared to the baseline in every visit. There was significant improvement in the doxofylline group compared to the other methylxanthines group at 2ndvisit and at the end of the study.

On analyzing the Spirometric parameter, Forced Vital Capacity, showed significant improvement in both the study groups compared to the baseline at every visit. There was no statistically significant difference in between the two groups throughout the study, indicating comparable efficacy.

The Spirometric parameter, forced expiratory Volume at the end of 1 second, revealed significant improvement in both the study groups compared to the baseline at every visit. There was no statistically significant difference in between the two groups throughout the study, indicating comparable efficacy.

On analyzing, the Peak Expiratory Flow Rate (PEFR), study revealed significant improvement in both the study groups compared to the baseline at every visit. There was no statistically significant difference in between the two groups throughout the study.

Comparing the FEV1/ FVC ratio in both the study groups revealed that there was no significant difference in between the groups throughout the study. There was statistically significant difference within the groups, compared to the base line at every visit.

In the present study, adverse drug reactions were noted in $10 \%$ and $28 \%$ of doxofylline and other methylxanthines group respectively. Incidence of overall adverse drug 
reactions were significantly more associated with Other methylxanthines; Group with $\mathrm{P}=0.041^{*}$. Among the adverse reactions, headache was the most common followed by nausea and insomnia.

Table 5: Comparison of peak expiratory flow rate in the two groups of patients studied.

\begin{tabular}{|llll|}
\hline & Doxofylline & Othermethylxanthines & P value \\
\hline Baseline & $4.95 \pm 0.93$ & $5.067 \pm 8.51$ & 0.317 \\
\hline$\%$ Predicted of baseline & $68.59 \pm 3.02$ & $68.96 \pm 1.63$ & 0.464 \\
\hline $1^{\text {st }}$ Visit & $5.16 \pm 0.96$ & $5.22 \pm 1.06$ & 0.770 \\
\hline$\%$ Predicted of $1^{\text {st }}$ Visit & $71.12 \pm 3.02$ & $71.31 \pm 1.62$ & 0.719 \\
\hline $2^{\text {nd }}$ Visit & $5.33 \pm 1.01$ & $5.35 \pm 1.12$ & 0.926 \\
\hline$\%$ Predicted of $2^{\text {nd }}$ Visit & $73.62 \pm 3.12$ & $73.26 \pm 1.57$ & 0.521 \\
\hline $3^{\text {rd }}$ Visit & $5.45 \pm 1.04$ & $5.49 \pm 1.13$ & 0.862 \\
\hline$\%$ Predicted of $3^{\text {rd }}$ Visit & $75.3 \pm 3.21$ & $75.10 \pm 1.53$ & 0.731 \\
\hline Significance from base line & & & - \\
\hline $1^{\text {st }}$ Visit & $\Delta=0.21 ; \mathrm{P}<0.001^{* *}$ & $\Delta=0.15 ; \mathrm{P}=0.001^{* *}$ & - \\
\hline $2^{\text {nd }}$ Visit & $\Delta=0.38 ; \mathrm{P}<0.001^{* *}$ & $\Delta=0.37 ; \mathrm{P}<0.001^{* *}$ & - \\
\hline $3^{\text {rd }}$ Visit & $\Delta=0.497 ; \mathrm{P}<0.001^{* *}$ & $\Delta=0.43 ; \mathrm{P}<0.001^{* *}$ & \\
\hline
\end{tabular}

Table 6: Comparison of FEV1/ FVC in the two groups studied.

\begin{tabular}{|llll|} 
& Doxofylline & Othermethylxanthines & P value \\
\hline Baseline & $67.11 \pm 2.32$ & $67.70 \pm 1.48$ & 0.155 \\
\hline $1^{\text {st }}$ Visit & $70.48 \pm 2.08$ & $70.21 \pm 1.99$ & 0.545 \\
\hline $2^{\text {nd }}$ Visit & $73.7 \pm 2.18$ & $73.07 \pm 1.95$ & 0.167 \\
\hline $3^{\text {rd }}$ Visit & $76.86 \pm 2.82$ & $77.35 \pm 2.26$ & 0.383 \\
\hline Significance from base line & & & \\
\hline $1^{\text {st }}$ Visit & $\Delta=3.36 ; \mathrm{P}<0.001^{* *}$ & $\Delta=2.36 ; \mathrm{P}=0.001^{* *}$ & - \\
\hline $2^{\text {nd }}$ Visit & $\Delta=6.69 ; \mathrm{P}<0.001^{* *}$ & $\Delta=5.22 ; \mathrm{P}<0.001^{* *}$ & - \\
\hline $3^{\text {rd }}$ Visit & $\Delta=9.74 ; \mathrm{P}<0.001^{* *}$ & $\Delta=9.50 ; \mathrm{P}<0.001^{* *}$ & - \\
\hline
\end{tabular}

Table 7: Comparison of overall tolerability parameters (adverse drug reactions) in the two groups of patients studied.

\begin{tabular}{|l|llll|}
\hline \multirow{2}{*}{} & \multicolumn{2}{c}{ Doxofylline } & \multicolumn{2}{c|}{ Othermethylxanthines } \\
\cline { 2 - 5 } & No & \% & No & \% \\
\hline Present & 5 & 16.67 & 14 & 46.67 \\
\hline Absent & 25 & 83.33 & 16 & 53.33 \\
\hline
\end{tabular}

\section{DISCUSSION}

The present comparative study on efficacy and tolerability of doxofylline over other methylxanthines involving 60 patients diagnosed with mild to moderate persistent bronchial asthma as per the may2014 guidelines of Global strategy for diagnosis and prevention of bronchial asthma updated, who had given written informed consent were enrolled for a study of 12 weeks duration. The study was conducted at chest hospital over a period of 8 months during which patients were evaluated and analyzed for epidemiological profile, disease spectrum, efficacy and tolerability parameters.

Maximum number of patients were between the age group 31 to 50 years with a mean age of 42 years in the doxofylline group and 38 years in other methylxanthines group. More than $96 \%$ of the study patients were from the urban region. Majority of the patients were females constituting $56 \%$ and $53 \%$ compared to males $43 \%$ and $46 \%$ in doxofylline and other methylxanthines group respectively as found in a study done by Aggarwal et al. ${ }^{5}$

\section{Efficacy parameters}

In the present study, asthma control test questionnaire revealed statistically significant improvement in both the groups compared to the baseline from 2nd visit onwards. There was statistically very significant difference in the doxofylline group $\left(\mathrm{p}=0.005^{*}\right.$ at the end of the study) compared to other methylxanthines group throughout the study. Thus, indicating better control of asthma in the 
doxofylline group with an increase in the score from the baseline to 2.34 as compared to 1.72 in the other methylxanthines group. This is in consistent with the study by patel $\mathrm{Y}$ et al, on controller medications as add on therapy to ICS and LABA for bronchial asthma management, that showed significant improvement in ACT score at end of the study compared to baseline using montelukast, doxofylline and increased dose of ICS, but no difference in between the study groups. ${ }^{6}$ ACT in particular has been found to be useful in the primary Health Care facilities in the developing countries. ${ }^{7}$

The subjective rating of asthma control in the study revealed statistically significant improvement of $15 \%$ at the end in both the groups compared to the baseline. There was significant improvement in the doxofylline group compared to the other methylxanthines group at $2^{\text {nd }}$ visit $(p=0.022)$ and at the end of the study.

There was statistically significant reduction at every visit, in the need for rescue medication ( $\beta 2$ agonist) in both the groups compared to the baseline. The reduction in the use of rescue medication compared to the baseline was 3.32puffs/day less in doxofylline group, as against reduction of 2.9 puffs/day less in other methyxanthines group by the end of the study $(\mathrm{p}=0.001 * *)$ suggesting better control in doxofylline compared to other methylxanthines group. Similar to a double blind randomized placebo controlled multicentric clinical trial conducted by Goldstein Mr et al, in 346 patients of chronic reversible bronchial asthma with four groups. Doxofylline $400 \mathrm{mg}$. Theophylline 250mg t.i.d, Doxofylline 200mg t.i.d, and Placebo group, demonstrated a significant reduction in the frequency of asthma attack and in use of albuterol with doxofylline $400 \mathrm{mg}$ t.i.d, as compared to that of theophylline $(\mathrm{p}<0.05){ }^{8}$

Spirometric analysis in this study revealed significant improvement in both the study groups compared to the baseline at every visit $(\mathrm{p}=0.001 * *)$. There was no statistically significant difference in between the two groups throughout the study. Compared to the baseline, percentage predicted of FVC improved by $4 \%$ and $3.5 \%$ FEV1, improved by $12.7 \%$ and $12.2 \%$, PEFR improved by $6.7 \%$ and $6.2 \%$ and FEV'1/FVC ratio improved by 9.7 and 9.5 in doxofylline and other methylxanthine groups respectively. Measurement of pulmonary function test in the placebo-controlled clinical trial by Goldstein and Chervinsky indicated that doxofylline $400 \mathrm{mg}$ t.i.d. is as effective as theophylline $250 \mathrm{mg}$ t.i.d. in improving the airflow obstruction, with significant increase in FEV1 (17\%) statistically significant improvement in both the groups compared to the placebo. In a randomized controlled trial by Dolcetti A et al, comparing doxofylline over placebo revealed significant improvement in FEV1, (>20\% after 2 hours) compared to baseline. The results in this study were in accordance to a double blind multicentre randomized trial, done by Melillo, $\mathrm{G}$ et al, that showed significant improvement in Spirometric parameters, reduced salbutamol consumption with doxofylline and Theophylline. ${ }^{9}$

\section{Tolerability parameters}

Tolerability of methylxanthines has always been an issue due to which, they have a consistent limitation in use, a newer methylxanthine with less adverse effect is in constant search in our study, a novel methylxanthine, doxofylline revealed significantly lower incidence of overall adverse drug reactions compared with other methylxanthines group with $\mathrm{P}=0.041 *$. Adverse drug reactions were noted in $10 \%$ of doxofylline and $28 \%$ in other methylxanthines group. Among the adverse reactions noted, headache was the most common followed by nausea and insomnia. Adverse events like vomiting epigastric pain, palpitation and tachycardia was seen only in other methylxanthines group, nervousness was seen in both the groups.

Clinical trials have shown a decrease in the incidence of adverse effects with doxofylline in comparison with those observed with theophylline, demonstrating an increased safety. Doxofylline has shown a better tolerability than theophylline and aminophylline in a study by Bossi. F et al, the probable reasons for better tolerability with doxofylline, could be due to low affinity for adenosine receptors (A1 and A2), it does not interfere with calcium flux through the cellular membrane nor antagonizes calcium channel antagonists that differentiate it from theophylline. ${ }^{10}$ The effective therapeutic dose of doxofylline has less cardio stimulant effect than theophylline, which allows its use without significantly increasing the cardiac frequency without arrhythmogenic effect. $^{11}$

It has also been demonstrated that Theophylline interferes with gastric secretion and gastrointestinal (GIT) motility, increasing the secretion of hydrochloric acid and pepsin in the stomach and reducing the pressure in the inferior esophageal sphincter, facilitating the development of gastroesophagic reflux. Doxofylline, on the other hand, does not increase the acid or pepsin output, reflected in the occurrence of fewer GI side effects. It has been suggested that this would make doxofylline better tolerated than theophylline in patients with peptic ulcer disease. ${ }^{12}$

Theophylline produces alterations in the structure and quality of sleep causing insomnia and many awakenings. In a study by Sacco et al, the number of arousals per night when treated with theophylline was almost double compared to when the subjects did not receive any medication, doxofylline did not result in more arousals. All subjects experienced REM sleep (Rapid Eye Movement) with doxofylline, then when treated with theophylline where only $50 \%$ of the subjects experienced REM sleep. This reduction in the quality of sleep does not occur among patients treated with doxofylline, who in addition experienced less restlessness. On comparing the recommended dose of doxofylline and its maximum dose, 
better tolerability was seen with doxofylline than with theophylline. ${ }^{13}$

\section{ACKNOWLEDGEMENTS}

Authors would like to thank the doctors from TB and Chest Hospital, Warangal, India. Authors would be failing in this duty if they do not thank all their patients included in this study.

Funding: No funding sources Conflict of interest: None declared

Ethical approval: The study was approved by the Institutional Ethics Committee of Kakatiya Medical College, Warangal, India

\section{REFERENCES}

1. Global strategy for asthma management and prevention, global initiative for asthma (GINA) revised asthma guidelines; May 2014. Available at: http://www.ginaasthma.org. Accessed 2nd July 2015

2. Rai SP, Patil AP, Vardhan V, Marwah V, Pethe M. Pandey IM Best Treatment Guidelines for bronchial asthma MJAFI. 2007:63:264-8.

3. Goodman and gilman's the Pharmacological basis of therapeutics $12^{\text {th }}$ Ed; Mc graw hill Newyork; 2011:031-1067.

4. Bar ne PJ. Pauwels RA. Theophylline in the management of asthma, time for appraisal? Eur Respir J. 1994:7:579-91.

5. Aggarwal AN, Chaudhry K, Chhabra SK, D Souza GA, Gupta D, Jindal SK, et al. Prevalence and risk factors for bronchial asthma in Indian adults: a multicentre study. Indian Journal of Chest Diseases and Allied Sciences. 2006 Jan 18;48(1):13.

6. Patel YA, Patel P, Bavadia H, Dave J, Tripathi CB. A randomized, open labeled comparative study to assess the efficacy and safety of controller medications as add on to inhaled corticosteroid and long - acting $\beta 2$ agonist in the treatment of moderate - to - severe persistent asthma. J Postgradu Med. OctoberDecember 2010:56:270-4.

7. Lai CK, Ko FW, Bhome A, De Guia TS, Wong GW, Zainudin BM, et al. Relationship between asthma control status, the Asthma Control Test ${ }^{\mathrm{TM}}$ and urgent health-care utilization in Asia. Respirology. 2011 May;16(4):688-97.

8. Dolcetti A, Osella D, de Fillippis G. Comparison of intravenously administered doxofylline and placebo for the treatment of severe acute airways obstruction. J Int Med Res. 1988:16:264-9.

9. Melillo G, Balzano G, Jodice F. Treatment of reversible chronic airways obstruction with doxofylline compared with slow - release theophylline. A double blind randomized multicentre trail, Int J clin Pharm Res. 1989:9;397-405.

10. Bossi R, Berni F. A double - blind multi center trail on efficacy and tolerability of doxofylline vs aminophylline in patients with chronic airway obstruction and reversible broncospasm. Ital $\mathrm{J}$ Che Disea. 1989:43:355-60.

11. Dini FL, Chronotropic and arrhythmogenic effects of two methylxanthine bronchodilators, doxofylline and theophylline, evaluated by Holter Monitoring Curr Ther Res. 1991:49:978-84.

12. Lazzaroni M, Grossi E, Porro GB. The effect of intravenous doxofylline or aminophylline on gastric secretion in duodenal ulcer patients. Alimentary pharmacology \& therapeutics. 1990 Dec;4(6):643-9.

13. Sacco C, Braghiroli A, GrossiE, Donner CF. The effects of doxofylline versus theophylline on sleep architecture in COPD pts. Monaldi Arch chest Dis. 1995;50;98-103.

Cite this article as: Alladi SK, Khan I. A comparative study of efficacy and tolerability of doxofylline over other methylxanthenes in mild to moderate persistent bronchial asthma patients in a tertiary care hospital. Int J Basic Clin Pharmacol 2018;7:1775-81. 